\title{
The fungal gene cluster for biosynthesis of the antibacterial agent viriditoxin
}

\author{
Andrew S. Urquhart ${ }^{1 \dagger}$, Jinyu $\mathrm{Hu}^{2 \dagger}$, Yit-Heng Choo ${ }^{2^{*}}$ and Alexander Idnurm ${ }^{1 *}$ (D)
}

\begin{abstract}
Background: Viriditoxin is one of the 'classical' secondary metabolites produced by fungi and that has antibacterial and other activities; however, the mechanism of its biosynthesis has remained unknown.

Results: Here, a gene cluster ( $v d t$ ) responsible for viriditoxin synthesis was identified, via a bioinformatics analysis of the genomes of Paecilomyces variotii and Aspergillus viridinutans that both are viriditoxin producers. The function of the eight-membered gene cluster of $P$. variotii was characterized by targeted gene disruptions, revealing the roles of each gene in the synthesis of this molecule and establishing its biosynthetic pathway, which includes a Baeyer-Villiger monooxygenase catalyzed reaction. Additionally, a predicted catalytically-inactive hydrolase was identified as being required for the stereoselective biosynthesis of $(M)$-viriditoxin. The subcellular localizations of two proteins ( $\mathrm{VdtA}$ and $\mathrm{VdtG}$ ) were determined by fusing these proteins to green fluorescent protein, to establish that at least two intracellular structures are involved in the compartmentalization of the synthesis steps of this metabolite.

Conclusions: The predicted pathway for the synthesis of viriditoxin was established by a combination of genomics, bioinformatics, gene disruption and chemical analysis processes. Hence, this work reveals the basis for the synthesis of an understudied class of fungal secondary metabolites and provides a new model species for understanding the synthesis of biaryl compounds with a chiral axis.
\end{abstract}

Keywords: Atropisomer, Eurotiales, Gene cluster, Laccase, Polyketide synthase

\section{Background}

Fungi produce a diverse array of polyketide-derived biaryl compounds with biological activities that are of interest as pharmaceutical lead molecules or through modulating fungal interactions with other species in the environment. Viriditoxin (Compound 1, Fig. 1) is a naphtho- $\alpha$-pyrone produced by the Eurotiales fungi Aspergillus viridinutans and Paecilomyces variotii, and in limited amounts by Aspergillus brevipes [1-3]. Related biaryl molecules have also been identified from other fungi including Fusarium spp. (aurofusarin [4]), Penicillium sp. (rugulotrosin A and B [5]) and Parastagonospora nodorum (elsinochrome A [6]) (these structures are

\footnotetext{
${ }^{*}$ Correspondence: yitheng.chooi@uwa.edu.au;

alexander.idnurm@unimelb.edu.au

${ }^{\dagger}$ Andrew S. Urquhart and Jinyu Hu have contributed equally to this work

${ }^{1}$ School of BioSciences, University of Melbourne, Melbourne, Australia

${ }^{2}$ School of Molecular Sciences, University of Western Australia, Perth, Australia
}

provided for comparison in Fig. 1), or Cercospora nicotianae (cercosporin [7]), A. niger kotanin [8], and Emericella desertorum desertorin [9]. The gene clusters for aurofusarin [10], elsinochrome [6,11], cercosporin [12, 13], kotanin and desertorin [14] have been identified.

Viriditoxin 1 exhibits interesting biological activities. In particular, viriditoxin was identified as a potent inhibitor of bacterial FtsZ, a protein that is required for bacterial cell division [15]. Subsequent work demonstrated that this was due to decreased transmembrane potential and perturbing membrane permeability, which prevents membrane proteins including FtsZ from effectively binding to the membrane [16]. Viriditoxin is also active against cancer cell lines and is a proposed starting molecule for possible therapeutic applications $[17,18]$. It has also been examined for a potential role in protection of sheep against blowflies [19]. In natural settings viriditoxin likely plays a role in competition against other microbes. For example, a strain of $P$. variotii was isolated 
<smiles>COC(=O)C[C@H]1Cc2cc3cc4c(c(O)c3c(O)c2c2c(OC)cc(O)c(OC)c2c2c(O)cc(O)c(OC)c42)C(=O)O1</smiles>

(M)-viriditoxin, 1<smiles>COC(=O)[C@]12Oc3cc(C)c(-c4c(C)cc5c(c4O)C(=O)C4=C(O)CC[C@@H](O)[C@@]4(OC(C)=O)O5)c(O)c3C(=O)C1=C(O)CC[C@H]2O</smiles>

rugulotrosin $\mathrm{A}$

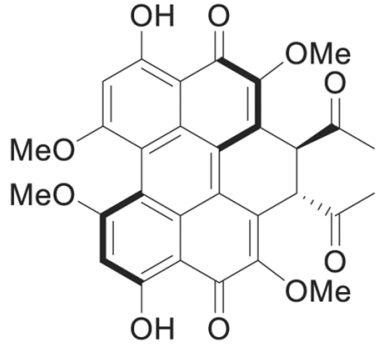

elsinochrome $\mathrm{A}$<smiles></smiles>

$(P)$-viriditoxin, $1^{\prime}$<smiles>COc1cc2c(c(O)c1-c1c(C)cc(O)c3c1O[C@@]1(OC)C(=C(O)CC[C@H]1O)C3=O)C(=O)C1=C(O)CC[C@@H](O)[C@]12OC</smiles>

rugulotrosin B<smiles>CCc1cc(=O)c2c(O)c3c(cc2o1)C(=O)C(OC)=C(C1=C(OC)C(=O)c2cc4oc(C)cc(=O)c4c(O)c2C1=O)C3=O</smiles>

aurofusarin

Fig. 1 Structures of $(M)$-viriditoxin and $(P)$-viriditoxin ( $\mathbf{1}$ and $\mathbf{1}^{\prime}$ ), as well as related metabolites produced by other fungi. The " $M$ " and " $P$ " refer to the atropisomer confirmations, with the helical configurations illustrated by the bold lines. $\mathbf{1}$ is the major form produced by $A$. viridinutans and $P$. variotii. Carbon numbers C3, C4 and C7 are indicated

as an endophyte from mangroves, and its antagonistic activities against bacteria attributed to production of viriditoxin [20].

From the perspective of biochemical synthesis, viriditoxin is of interest as it is a biaryl compound with axial chirality, in which rotation about the biaryl axis is hindered, resulting in two different stable forms that are known as atropisomers. The two atropisomers are referred to as $M$-viriditoxin $\mathbf{1}$ and $P$-viriditoxin $\mathbf{1}^{\prime}$ with the $M$ stereoisomer $\mathbf{1}$ being the major natural product found in P. variotii and A. viridinutans ([21], Fig. 1). The formation of such compounds requires the coupling of two subunits. How this coupling occurs in fungi is beginning to be understood, as both P450 monooxygenases and laccases/multicopper oxidases are able to catalyze this coupling. While P450 monooxygenases have been shown to be capable of stereoselective coupling [14], none of the fungal laccase/multicopper oxidases enzymes that have been implicated in biaryl coupling have demonstrated stereospecificity [4, 22, 23]. In plants, laccases are able to catalyze stereoselective coupling in combination with dirigent proteins; however, no such proteins have been reported in fungi [24]. Despite the growing appreciation of the importance of atropisomers in the pharmaceutical industry, it is still not understood how laccase-catalyzed reactions can result in stereoselective biosynthesis of biaryl compounds in fungi $[25,26]$.

The rationale of this study was to identify the genetic components responsible for synthesis of viriditoxin and to establish how this molecule is synthesized by the construction and analysis of metabolites produced in mutant strains in those genes. The genomes of two isolates of $P$. variotii were recently sequenced [27], opening opportunities for identifying the basis for the synthesis of viriditoxin, or other metabolites that this fungus may produce. As part of this work we generated a draft genome of the original isolate that produces viriditoxin, A. viridinutans, and find related gene clusters in these two Eurotiales species. Gene manipulation tools are not yet available for $A$. viridinutans. Hence, the gene cluster was functionally characterized in $P$. variotii, a newly-emerging model fungal species where methods for gene manipulation were recently developed [27].

\section{Results and discussion}

Identification of a putative cluster of genes for viriditoxin biosynthesis by comparative genomics between $P$. variotii and $A$. viridinutans

We recently sequenced the genome of $P$. variotii CBS 101075 , which is known to produce viriditoxin [2] and a 
second $P$. variotii strain, CBS 144490 [27]. For comparison, the $A$. viridinutans strain FRR 0576 genome was sequenced using Illumina HiSeq2500 paired-end reads. $14,757,842$ reads were generated and assembled into 285 contigs, totaling approximately $30 \mathrm{Mb}$ (Additional file 1: Table S1). The assembly and raw reads are available from GenBank under BioProject PRJNA513223. A curious commonality between $A$. viridinutans and $P$. variotii is that both genomes have a bimodal GC and AT DNA composition, of which the AT-rich regions of $P$. variotii have been attributed to the action of active repeat inducted point (RIP) mutation [27].

The naphthopyrone scaffold of viriditoxin is similar to a number of other fungal polyketide secondary metabolites, such as aurofusarin or the $\alpha$-pyrone elsinochrome $\mathrm{C}$, whose synthesis requires polyketide synthase (PKS) enzymes. Hence, candidate genes encoding PKSs were sought first in the two $P$. variotii genomes using BLAST with the full length protein sequences of the PKS enzymes PKS12 (which is required for aurofusarin biosynthesis [28]), or ElcA (which is required for elsinochrome $\mathrm{C}$ biosynthesis [11]). Matches were obtained to 10 putative PKS-encoding genes in each strain. Two iterative type I PKS enzymes with low expect values ( $E$-value 0.0 , vs. next best $E$-value $3 \mathrm{e}^{-65}$ ) were identified: protein IDs 480069 (named VdtA) and 456077 (named PvpP) in the CBS 101075 genome, available through MycoCosm $[27,29]$.

BLAST was used to identify the homologous genes for these two $P$. variotii PKS enzymes and the genes on either side of them in the assembled $A$. viridinutans genome sequence. This approach revealed a putative candidate cluster for viriditoxin (hereafter referred to as the $v d t$ cluster) common to both species. This cluster consists of nine genes in $P$. variotii and eight in A. viridinutans, with the difference being due to the presence of a second putative PKS-encoding gene $(v d t X)$ in $P$. variotii (Fig. 2a, Additional file 1: Table S2). The predicted functions of these proteins are presented in Additional file 1: Table S2.

While performing this genome sequencing and analysis, an independent genomic analysis was conducted by Fürtges et al. who proposed the same biosynthetic cluster in A. viridinutans, and demonstrated dimerization activity of the recombinantly-expressed laccase, albeit on a non-native substrate [22]. Further, a gene cluster was identified for the synthesis of agnestins A and B in P. variotii [30].

\section{Transcription factor VdtR regulates expression of the $v d t$ gene cluster and viriditoxin synthesis}

Fungal secondary metabolite clusters may contain a transcription factor responsible for regulation of the cluster genes [31]. VdtR has the characteristics of a six-cysteine
(C6) zinc cluster type of transcription factor, with similarity to AflR that regulates the genes in the aflatoxin/ sterigmatocystin biosynthesis gene cluster in Aspergillus species. The $v d t R$ gene was deleted by replacing most of the open reading frame with a construct conferring resistance to hygromycin via homologous recombination. The expression of the genes in the gene cluster was compared between the wild type and $v d t R$ deletion strains by quantitative PCR. This revealed reduced transcript levels of genes in the $v d t$ cluster when $v d t R$ was deleted (Fig. 2b), confirming the identity of VdtR as a regulator of the genes in the cluster. The $v d t R$ deletion strain also lost the ability to synthesize viriditoxin (Fig. 3). Characterization of the transcript levels of the genes flanking the putative gene cluster in the $P$. variotii wild type and $v d t R$ strains defines the boundaries of the cluster: the flanking genes encoding proteins ID510298, ID480071 and ID423248 were unaffected (gene ID420046 could not be detected; Fig. 3b).

\section{The polyketide synthase VdtA is required for the first step of viriditoxin biosynthesis}

$P$. variotii encodes two candidate $\mathrm{PKSs}$ that could be involved in the synthesis of viriditoxin, i.e. ID480069 (named VdtA) and ID456077 (named PvpP). The majority of each PKS gene was replaced with a hygromycin resistance marker, through homologous recombination of constructs transformed into P. variotii using Agrobacterium-mediated transformation, and the mutant strains were tested for viriditoxin synthesis.

The deletion of $v d t A$, in both $P$. variotii strains CBS 101075 and CBS 144490, abolished the production of 1 and related derivatives $\mathbf{2}$ and $\mathbf{3}$ in the culture filtrates of the strains, as detected by liquid chromatography with photodiode array detection-mass spectrometry (LCDAD-MS), confirming the role of VdtA early in the biosynthesis pathway of viriditoxin (Fig. 3). Structures of these three compounds were confirmed by 1D and 2D nuclear magnetic resonance (NMR) analysis. Electronic circular dichroism (ECM) spectroscopy was used to determine the helical conformation of the compounds by comparing to a commercial standard of 1 (Additional file 1: Tables S3, S5, S6, Figures S3-S8, S15-S26).

In contrast to the impact of deletion of $v d t A$, mutation of the second candidate gene for viriditoxin synthesis that was identified by bioinformatics approaches did not impact viriditoxin production (Fig. 3a). Instead, deletion of the $p v p P$ gene in strain CBS 101075 altered the pigmentation of the mutant strains (Fig. 3b). PvpP is homologous to PksP of Aspergillus fumigatus (GenBank accession Q4WZA8), which is required for formation of the pigment DHN melanin [32]. PksP of A. fumigatus is responsible for the synthesis of the yellow molecule 


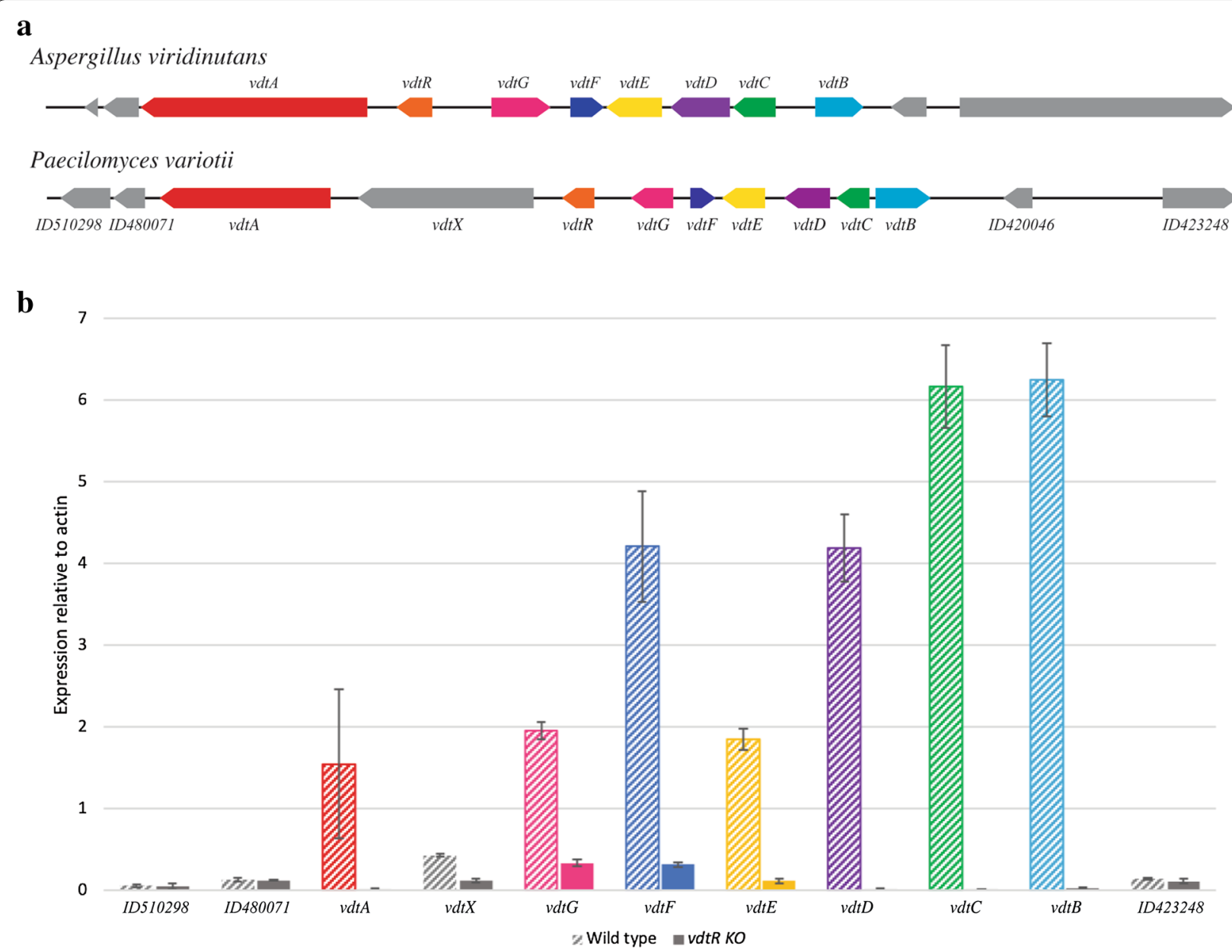

Fig. 2 Putative $v d t$ gene cluster encoding enzymes for viriditoxin 1 biosynthesis. a Comparison between the A. viridinutans and P. variotii clusters; predicted functions of the encoded proteins are presented in Additional file 1: Table S2. b Comparison of the transcript levels of $v d t$ genes and those flanking the cluster between wild type and $v d t R$ deletion mutant measured by $\mathrm{qPCR}$

YWA1. BLAST with DHN melanin pathway components in A. fumigatus (i.e. GenBank accessions E9QUT3, Q4WZB3 and Q4WZB4) [33, 34] required to convert YWA1 into other pigments revealed their absence from the $P$. variotii genome. Thus, PvpP of $P$. variotii is required for the synthesis of the characteristic yellow pigment observed for petri dish cultures, and analysis of the genome sequence supports the hypothesis that this pigmentation is due to the synthesis of YWA1 or a related molecule, without the additional modifications seen in other Eurotiales species.

Different polyketide synthase reactions of VdtA and PvpP are further supported by the analysis of their Product Template domains. Liu et al. defined these domains from fungal proteins into eight groups, based on phylogenetic analyses, and correlated their classification with the enzyme first ring cyclization modes [35]. VdtA matches group IV (C4-C9 cyclization) and PvpP matches group
III (C2-C7 cyclization), which is consistent with the structures of viriditoxin or the putative YWA1-related pigment, respectively.

The $v d t$ gene cluster in $P$ variotii encodes a second putative PKS that is absent from the $A$. viridinutans gene cluster. Hence, one hypothesis is that this gene is not required for viriditoxin production. Disruption of this gene $(v d t X)$ via homologous recombination was unsuccessful, so we employed an alternative approach using RIP, which is an active process in $P$. variotii during heterothallic sexual crosses [27]. RIP is a fungal-specific mechanism in which duplicated DNA is recognized and specifically mutated as part of the sexual cycle, and has been used to make targeted mutations in genes in $\mathrm{Neu}$ rospora crassa [36]. To achieve this, a second partial copy of $v d t X$ was introduced into $P$. variotii CBS 101075, and that strain crossed to the wild type strain of opposite mating type (CBS 101075) such that RIP could mutate 

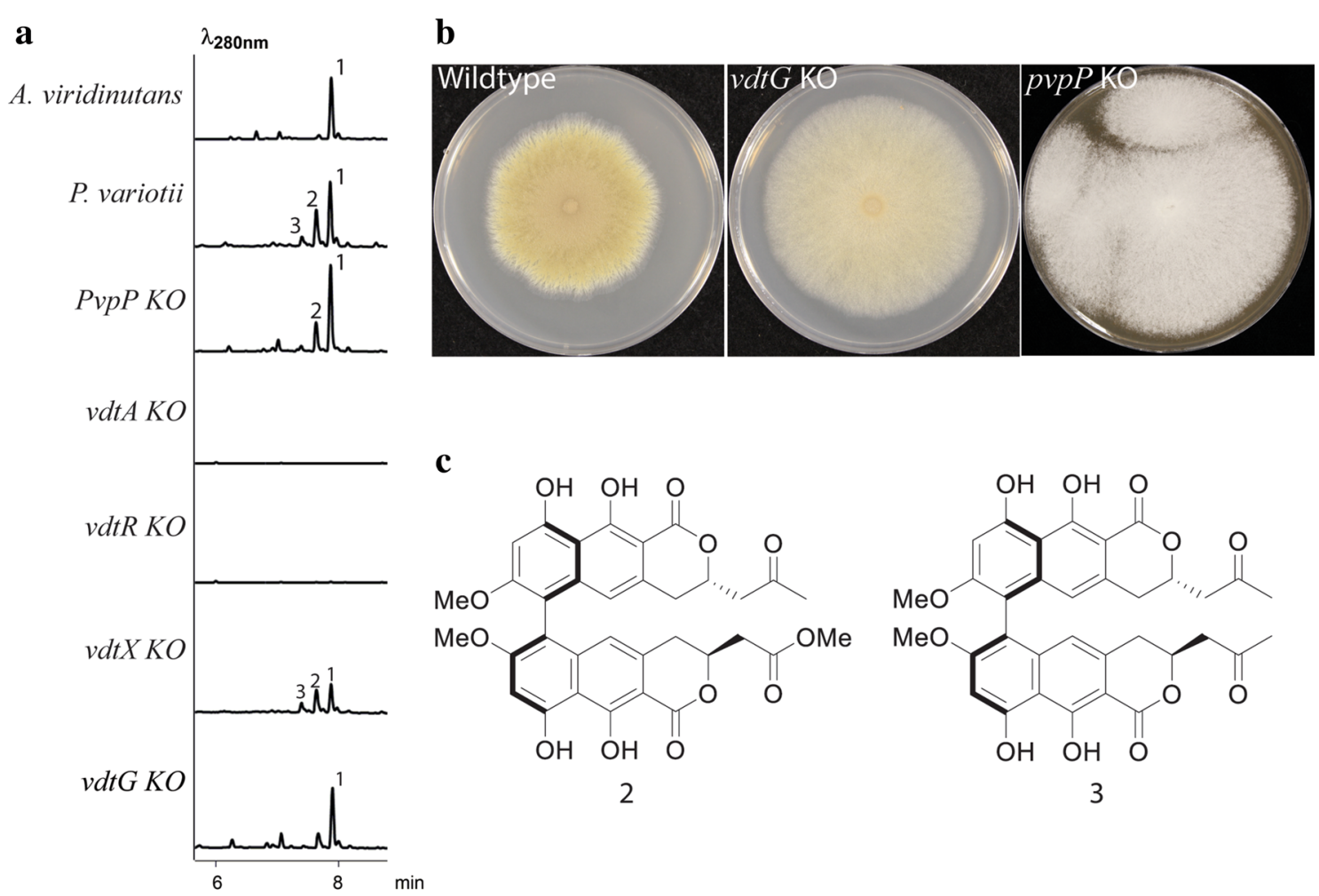<smiles>COC(=O)C[C@H]1Cc2cc3c(-c4c(OC)cc(O)c5c(O)c6c(c(O)c45)C(=O)O[C@@H](CC(C)=O)C6)c(O)cc(O)c3cc2C(=O)O1</smiles>

2<smiles>COc1cc(O)c2c(O)c3c(cc2c1-c1c(OC)cc(O)c2c(O)c4c(cc12)C[C@H](CC(C)=O)OC4=O)C[C@@H](CC(C)=O)OC3=O</smiles>

3

Fig. 3 a LC-DAD-MS analysis of A. viridinutans and P. variotii CBS 144490 and $P$. variotii mutants ( $p v p P, v d t A, v d t R$, $v d t X$ and $v d t G)$. b Deletion of $v d t G$ resulted in a sporulation defects and deletion of pvpP resulted in a loss of conidial pigmentation. $\mathbf{c}$ Structures of the additional viriditoxin derivatives (2 and $\mathbf{3}$ ) produced by the wild type P. variotii strain CBS 144490

the duplicated DNA, followed by the identification of progeny carrying mutations. One progeny was obtained in which the native copy of the gene had been heavily mutated by RIP, introducing premature stop codons (Additional file 1: Figure S1). The ability to generate mutants efficiently through RIP adds another technique to the repertoire available in $P$. variotii.

The $v d t X$ RIP mutant continued to produce viriditoxin, indicating that $\mathrm{VdtX}$ is not required for viriditoxin biosynthesis (Fig. 3a). The reduction in the amount of metabolites produced in the RIP mutant compared to the wild type might be due to the variability expected in the progeny of a sexual cross. As $v d t X$ is not involved in viriditoxin synthesis, the gene was not considered further, but might possibly play a role in the production of additional metabolites not extracted or detected by the techniques employed in this study.

\section{The polyketide synthase VdtA is localized} within a specialized structure

The localization of PKS enzymes in fungal secondary metabolite synthesis is largely unknown. The PksP (Alb1) PKS of Aspergillus species that is involved in the production of melanin is localized to the endosome [37], and it represents the only fungal PKS with a demonstrated subcellular localization. A strain expressing a VdtA-GFP fusion protein displayed green fluorescence tightly confined to small circular structures within the cells (Fig. 4a). Expression of GFP alone using PLAU17 [38] localizes to the cytosol (data not shown). The VdtA-GFP strain continued to produce viriditoxin, indicating that the addition of GFP to its carboxyl-terminus end did not destroy the activity of the enzyme (data not shown) and that the structures are not an artefact from gene manipulation. These small spherical structures are reminiscent of peroxisomes. Peroxisomes have been implicated in the production of other fungal secondary metabolites [39]. In particular, the aflatoxin precursor norsolorinic acid, which is produced by PksA, is localized to the peroxisome [40]. However, the PksA enzyme itself has not been localized. Given sequence similarity between the aflatoxinproducing PksA and P. variotii VdtA, we explored the possibility that the unusual localization pattern observed for VdtA-GFP represented peroxisome localization by dual-labelling with an mCherry-SKL peroxisomal construct. The addition of the three amino acid motif SKL 


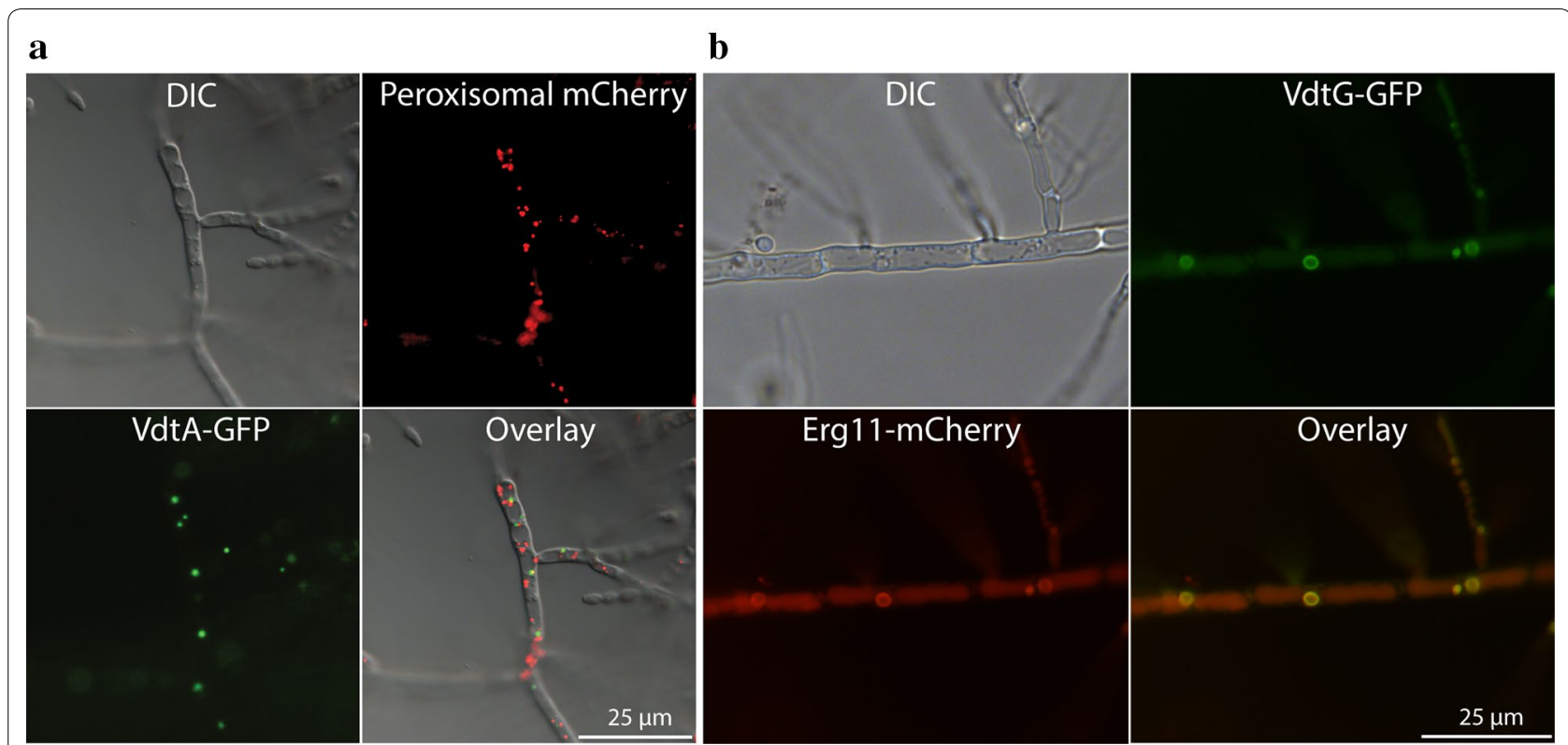

Fig. 4 Viriditoxin is synthesized in at least two different organelles. a VdtA-GFP fusion protein localizes to small spherical structures within hyphae, which are distinct from the peroxisomes (labelled with mCherry-SKL). $\mathbf{b}$ VdtG-GFP localized around a small circular structure and co-localizes with the endoplasmic reticulum marker Erg11-mCherry

to the carboxy terminus is widely used to target fluorescent proteins to the peroxisome [41]. However, VdtAGFP and mCherry-SKL showed clearly distinct patterns of localization. Based on previous analysis of nuclei and mitochondria using tagged proteins we can exclude those organelles [27]. Thus, these structures where VdtA is targeted remain unknown.

\section{The transporter VdtG is localized to an internal organelle and is not essential for viriditoxin production}

Deletion of the gene encoding a putative transporter VdtG had no effect on the production of viriditoxin or its precursors, although their levels were reduced compared to the wild type strain (Fig. 3a). However, the gene deletion strains have a sporulation defect (Fig. 3b). Production of secondary metabolites is often developmentally timed with the beginning of spore formation [42], so we hypothesize that this phenotype is likely to result from toxicity effects beginning at this stage. Such morphological differences have not been observed in previously disrupted transporters associated with secondary metabolite gene clusters such as the genes aflT in A. parasiticus [43] or sirA in Leptosphaeria maculans [44], although in the case of SirA, sensitivity to sirodesmin was increased in the $\operatorname{sir} A$ mutants.

To further probe the role of this putative transporter, DNA encoding GFP was fused to the native $v d t G$ gene of $P$. variotii. Strains expressing this fusion protein did not show the sporulation defects present in the $v d t G \mathrm{KO}$ strains, indicating that the fusion protein is functional (data not shown). Fluorescence microscopy revealed that VdtG is localized around an internal membrane structure and the septa (Fig. 4b). Taken together these results lead us to hypothesize that VdtG is required to compartmentalize the synthesis of viriditoxin or its intermediates within the cell to limit auto-toxic effects.

The VdtG-GFP structure resembles the 'toxisomes' of F. graminearum [45] and 'aflatoxisomes' of Aspergillus species [46]. Toxisomes are proliferations of the smooth endoplasmic reticulum [47]. For this reason, we examined the co-localization of VdtG-GFP with a known endoplasmic reticulum protein, Erg11 (Cyp51) required for sterol synthesis, as an mCherry fusion [48]. This showed clear co-localization of the two proteins suggesting that the VdtG-GFP structure is part of the endoplasmic reticulum or an endoplasmic reticulum derived structure (Fig. 4b). The localization of VdtG to these structures in $P$. variotii provides evidence that these structures might be homologous to toxisomes and aflatoxisomes. There is no evidence that the VdtG and VdtA structures co-localize, given that the structures to which VdtA are localized are more numerous and of different size.

\section{VdtC, VdtF and VdtE are involved in tailoring of the polyketide precursor produced by VdtA to the monomer semi-viriditoxin}

We next generated knockout mutants for the genes encoding putative 'tailoring' enzymes $\mathrm{VdtC}, \mathrm{VdtF}$ and 
VdtE. LC-DAD-MS analysis of the culture extracts revealed major changes in the metabolite profiles (Fig. 5). The compounds were purified from the individual mutants and subjected to NMR analysis for elucidation of the structures of 3, 4, 5, 7, 8 and 9 (Additional file 1: Tables S6-S11, Figures S21-S56) whereas 6 is proposed based on mass (Additional file 1: Figure S1). ECM spectroscopy was used to determine the helical confirmation of the compounds $3,4,5,7,8$ and 9 (Additional file 1: Tables S6-S11). The structures of these compounds provide insights into the function of each of these enzymes.

VdtC shows $44 \%$ similarity to the $O$-methyltranstrase AurJ in F. graminearum that is involved in the biosynthesis of aurofusarin [4]. As expected, deletion of $v d t C$ results in the production of compounds 4,5 and $\mathbf{6}$, which differ from viriditoxin in that the hydroxyl group (red $\mathrm{OH}$ on Fig. 5; off carbon 7 in Fig. 1) has not been methylated. Interestingly, circular dichroism data showed that the two metabolites purified in sufficient quantity for ECM analysis, $\mathbf{4}$ and 5, are both in the $P$ helical form, suggesting that the $O$-methyl group is important for the stereoselective coupling of the monomers.

BLAST of the VdtF protein sequence revealed a number of close fungal homologs, but also shows $29 \%$ identity to the 3-oxoacyl-[acyl-carrier-protein] reductase FabG of bacterium Eschericha coli and thus may be able to catalyze a reduction reaction [49]. Deletion of $v d t F$ results in the production of compounds $7, \mathbf{8}$ and $\mathbf{9}$, which differ from 1 in that the $\mathrm{C} 3-\mathrm{C} 4$ double bond has not been reduced (Fig. 5). We thus conclude that VdtF is responsible for the reduction of this bond.

BLAST against the Swiss-Prot database show that VdtE displays homology to a number of previously characterized Baeyer-Villiger monooxygenases (BVMOs). BVMOs are a group of enzymes responsible for the conversion of ketones into esters by inserting an oxygen atom between a carbonyl carbon and its neighboring carbon [50]. Consistent with the hypothesis that VdtE is a Baeyer-Villiger monooxygenase, deletion of $v d t E$ resulted in the accumulation of compound $\mathbf{3}$ in which the ketone group has not been converted into the methyl ester found in $\mathbf{1}$ (Fig. 5). The activity of VdtE is similar to MoxY of the aflatoxin biosynthesis cluster found in Aspergillus spp. [51]. MoxY converts the ketone hydroxyversicolorone ( $\mathrm{HVN}$ ) into the acetate ester versiconal hemiacetal acetate (VHA). This is a rather unique reaction, so efforts towards purification of recombinant $\mathrm{VdtE}$ are ongoing to characterize further this unusual enzyme.

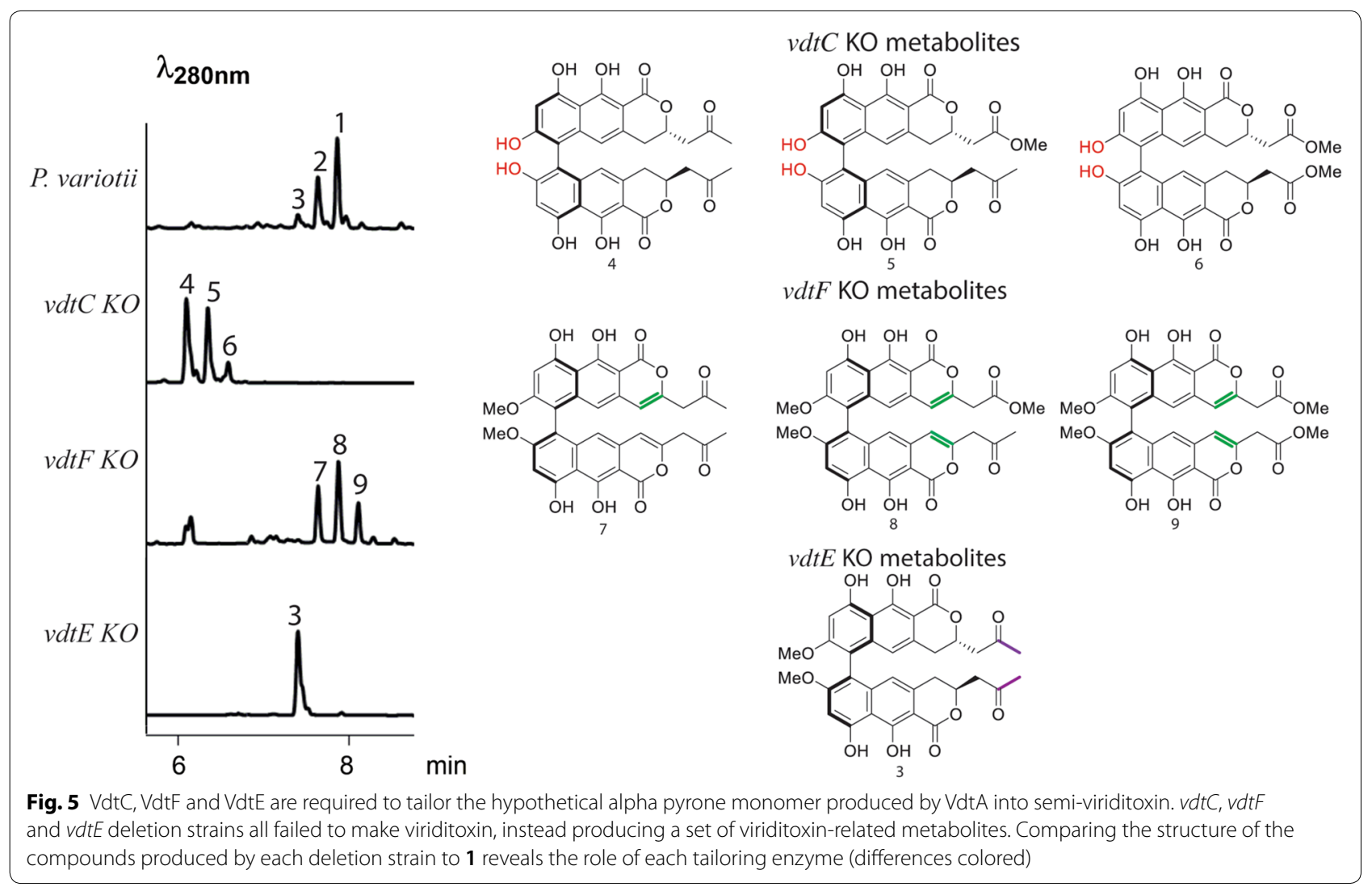


The laccase VdtB is involved in dimerization while a non-catalytic hydrolase-like protein VdtD affects the stereochemistical outcome of the coupling

$\mathrm{VdtB}$ is similar to laccase enzymes previously implicated in oxidative coupling, including GIP1 that is suggested to play a role in the dimerization of two rubrofusarin molecules to form aurofusarin in F. graminearum [4] and MCE that dimerizes monapinone A into dinapinone $A$ in Talaromyces pinophilus [23]. Thus, we hypothesized that $\mathrm{VdtB}$ would be required to form the biaryl bond between two semi-viriditoxin molecules to produce viriditoxin. Consistent with this hypothesis, disruption of the $v d t B$ gene led to the accumulation of four major compounds, in which their $m / z$, as detected by LC-MS, corresponded to the molecular weight expected for monomers (Fig. 6a, b; Additional file 1: Figure S1). The structure of three of these compounds 10, 11 and 12 is predicted based on mass. This confirms that the laccase VdtB is responsible for the dimerization step in the synthesis of viriditoxin.

The production of semi-viriditoxin $\mathbf{1 2}$ is interesting because this shows that the tailoring steps catalyzed by VdtC, VdtF and VdtE most likely to take place prior to dimerization. However, the possibility of these tailoring enzymes acting on dimers cannot be excluded. Furthermore, the fact that the $v d t C, v d t F$ and $v d t E$ deletion mutants produce dimerized compounds shows that VdtB is not strictly specific to semi-viriditoxin in vivo. Our findings concur with the results of a recent study that demonstrated that the VdtB homolog of $A$. viridinutans is able to dimerize the non-native substrate $(R)$-semi-vioxanthin, using heterologously-produced cell-free extracts from Aspergillus niger [22]. However, the heterologouslyproduced VdtB lacked the appropriate stereospecificity in an in vitro assay [22].

Bioinformatic analysis of VdtD shows it has amino acid sequence similarity to serine hydrolases (Fig. 6d). This is a functionally diverse group that includes catalyticallyactive hydrolases and other proteins that have evolved diverse functions that are independent of catalytic activity. Two such proteins that have lost catalytic function and taken on new roles are neuroligins, which are important components of the post-synaptic membrane [52], and thyroglobulin, a precursor of thyroid hormones [53]. The catalytically-active serine hydrolases share a nucleophilic serine residue contained within the conserved $\mathrm{G}-\mathrm{X}-\mathrm{S}-\mathrm{X}-\mathrm{G}$ motif (where $\mathrm{X}$ is any amino acid) at their

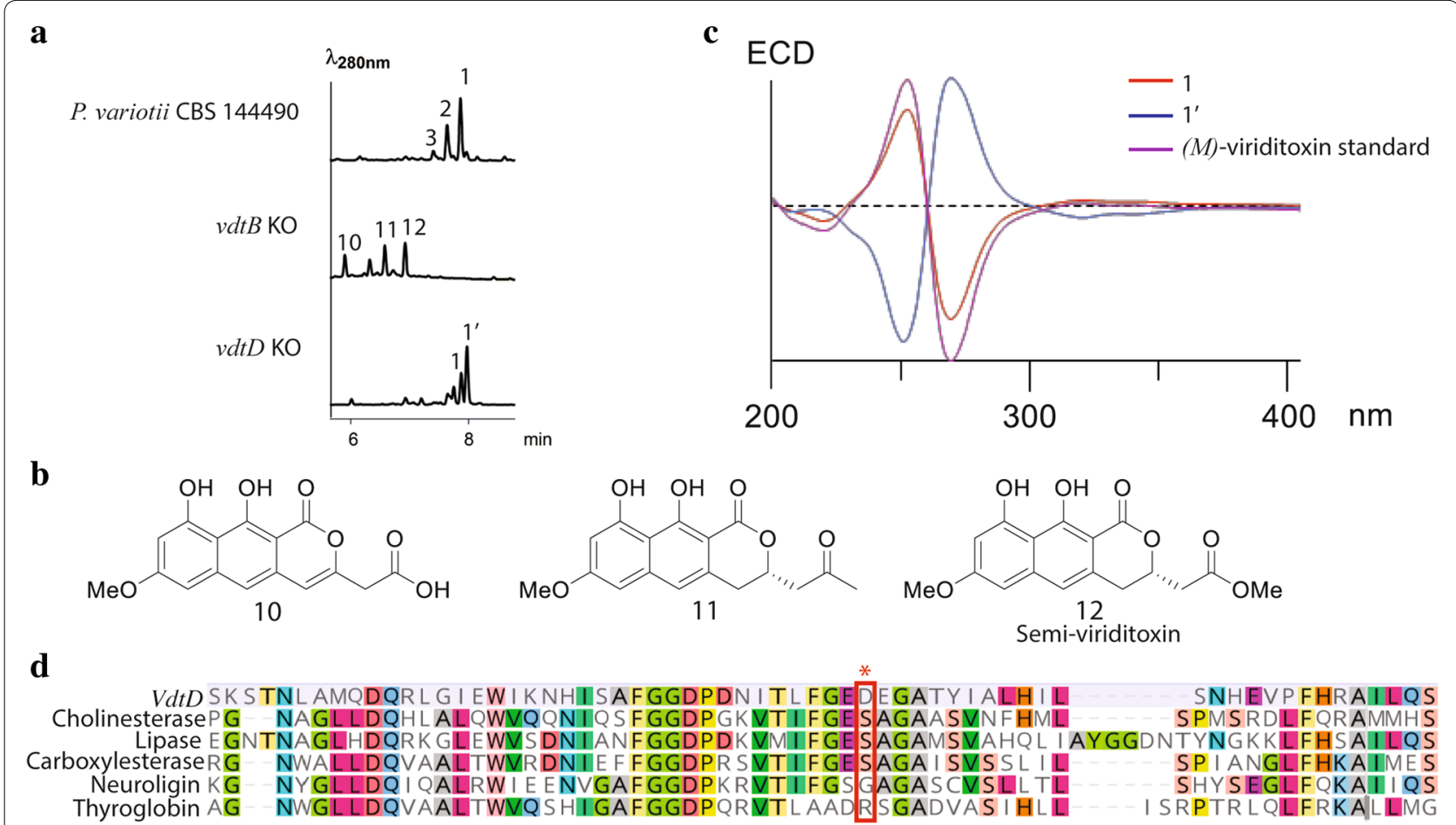

Fig. 6 a LC-DAD-MS analysis of the $v d t B$ and $v d t D$ deletion strains. $\mathbf{b}$ The predicted structures of monomers produced by the $v d t B$ deletion strain based on mass. c Comparison of the electronic circular dichroism data of $\mathbf{1}$ vs $\mathbf{1}^{\prime}$ showing that the spectra are mirrors of each other, indicative of two atropisomeric forms. d Multiple sequence alignment of VdtD and a lipase from Geotrichum candidum (UniProtKB/Swiss-Prot: P22394.2), a choline esterase from Branchiostoma lanceolatum (UniProtKB/Swiss-Prot: Q95000.1), a carboxylesterase from Felis catus (UniProtKB/Swiss-Prot: Q81034.1), and a neuroligin (UniProtKB/Swiss-Prot: Q8N0W4.1) and a thyroglobin (UniProtKB/Swiss-Prot: O08710.3) from Homo sapiens. Red box encloses the active site serine in the G-X-S-X-G motif of active hydrolases 
active site [54]. The non-catalytic variants lack this active site serine [55].

To explore if VdtD is likely to be an active esterase we compared the sequence of VdtD to the top 100 BLAST results in the Swiss-Prot database. These mostly include enzymes, however there are also some neuroligins and thyroglobulins. A sequence alignment of a subset of these revealed that, as expected, thyroglobin and neuroligins lacked the serine residue whereas the serine was present in proteins with enzymatic activity (Fig. 6d). Importantly, VdtD lacks this serine, having instead an aspartate in that position. This suggests that VdtD is probably not an active hydrolase, and as with its mammalian homologs neuroligin and thyroglobulin has taken on new roles.

Intriguingly, disruption of $v d t D$ alters the ratio of the two atropisomers of viriditoxin $\mathbf{1}$ versus $\mathbf{1}^{\prime}$ (Fig. 6a). While the wild-type strain strongly favors the production of the $M$ form $\mathbf{1}$, the $v d t D$ mutant produced favored the $P$ form $\mathbf{1}^{\prime}$, as revealed by circular dichroism (Fig. 6c). NMR data for $\mathbf{1}^{\prime}$ is provided in Additional file 1: Table S4 and Figures S9-S14. Despite the growing appreciation of the importance of atropisomeric compounds [26], how organisms selectively synthesize particular atropisomeric forms remains unknown. Given that the laccase VdtB alone has been shown to favor the unexpected $P$-configured stereoisomer in a heterologous system [22] we suggest VdtD is likely to be required to control the stereoselectivity of the laccase-catalyzed reaction, possibly playing an analogous role to the dirigent proteins of plants [24].

The stereochemistry of the metabolites produced by the tailoring-enzyme gene knockouts (Fig. 5) provides initial insight into the substrate requirements of the hypothesized VdtD/VdtB coupling system. The compounds produced by the $v d t F$ and $v d t E$ knockouts, lacking $\mathrm{C} 3-\mathrm{C} 4$ bond reduction and the methyl ester, respectively, are predominantly coupled in the $M$ helical conformation as $\mathbf{1}$. This suggests that these chemical groups are not integral for interaction with coupling enzymes. However, while not the dominant product as measured by ECM (Additional file 1: Table S6), some of the corresponding $P$ stereoisomer appears to form a shoulder peak in 3 (Fig. 3), so we cannot completely exclude the possibility that the methyl ester makes a contribution to the interaction. On the other hand, the metabolites produced by the $v d t C$ mutant that lack methylation of the oxygen attached to $\mathrm{C} 7$ have been coupled in the unexpected $P$ configuration. We hypothesize that interaction of this methyl group with either VdtB or VdtD is likely to be crucial for a controlled stereochemical outcome. Further biochemical studies will seek to establish the mechanism by which VdtD controls the stereospecificity of VdtB.

\section{Conclusion}

The gene cluster responsible for the production of viriditoxin has been identified in the genome of two Eurotiales species. First, knock-out mutations in $P$. variotii of all the enzymes required for the production of viriditoxin and a candidate transcription factor for their regulation allowed us to elucidate a predicted biosynthetic pathway of this helical chiral biaryl compound (Fig. 7). Second, we have also uncovered an intriguing cellular localization pattern for the PKS enzyme responsible for the first step in the pathway. Third, we have demonstrated that a likely catalytically inactive hydrolase enzyme is required for controlling atropisomeric conformations through an as yet undefined mechanism. Complementary biochemical analyses using heterologous expression of these enzymes have subsequently substantiated the steps in the pathway [56]. Future experiments will be able to provide additional resolution into the process of the synthesis of viriditoxin.

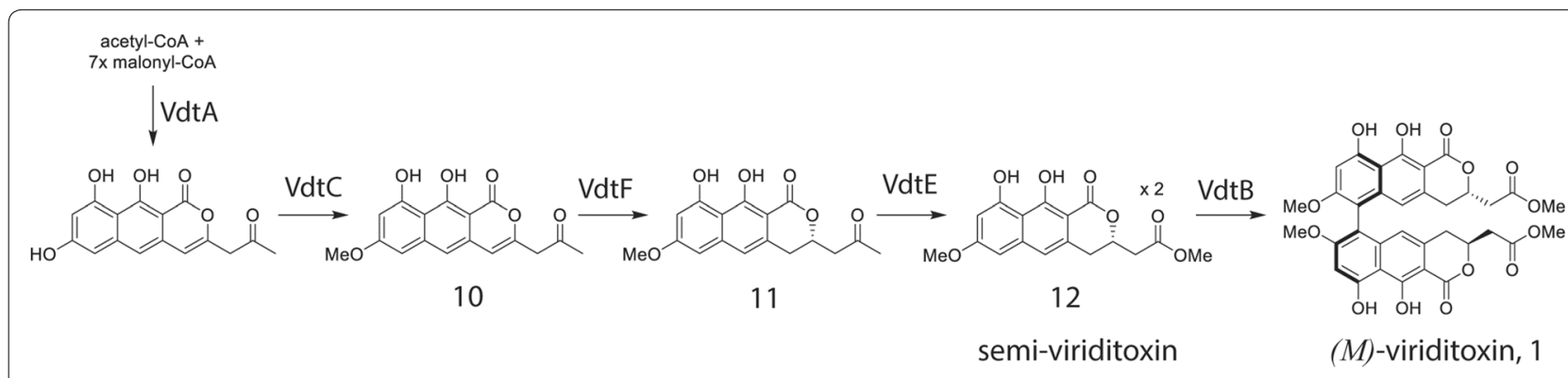

Fig. 7 A proposed pathway for the biosynthesis of viriditoxin as based on the metabolites identified in gene deletion strains of $P$. variotii. Note that the order in which the enzymes $\mathrm{VdtC}, \mathrm{VdtF}$ and $\mathrm{VdtE}$ act has not been determined 


\section{Materials and methods}

\section{Strains, DNA extraction, and DNA sequencing of $A$. viridinutans}

Two $P$. variotii strains used in this study were CBS 101075, which is the type strain of the teleomorph of P. variotii (Byssochlamys spectabilis), and CBS 144490, which we isolated previously [27]. The A. viridinutans ex-type strain FRR 0576 was used. Genomic DNA was extracted from 4-day old liquid cultures grown in potato dextrose broth (PDB) using an established method [57], followed by treatment with RNAseA. $A$. viridinutans was sequenced on an Illumina HiSeq2500 instrument using $125 \mathrm{bp}$ paired-end reads. The reads were assembled using Velvet [58]. Regions homologous to putative clusters in the $P$. variotii genome were identified by BLASTx searches against the $A$. viridinutans assembly.

\section{Gene expression analysis of the Vdt cluster via qPCR}

RNA was extracted from 3-day old cultures of $P$. variotii grown in PDB using Trizol reagent (Invitrogen) following the manufacturer's directions. cDNA was synthesized using AMV reverse transcriptase (New England Biolabs) following the standard protocol provided by the manufacturer. qPCR reactions were conducted using KAPA SYBR FAST qPCR Master Mix. The primer pairs used for each gene are given in Additional file 1: Table S12.

\section{Generation of gene knockout strains}

Gene knockout strains were generated by homologous recombination for each gene in the cluster to replace most of the open reading frames with a construct conferring resistance to hygromycin, with the exception of $v d t X$. The constructs were generated by first assembling two fragments corresponding to the upstream and downstream gene regions, into plasmid pPZP-201BK that was digested with EcoRI and HindIII, using Gibson Assembly with the addition of a restriction site between the two fragments. The HYG cassette on plasmid pMAI6 was amplified by PCR and introduced into this restriction site using a second Gibson reaction. Primers used to amplify the HYG cassette with appropriate homology to introduce it into each construct are given in Additional file 1: Table S12.

The constructs were introduced into $P$. variotii strain CBS 144490 or CBS 101075 via Agrobacterium tumefaciens mediated transformation (AtMT) as described previously [27]. Successful gene replacement events were identified via PCR using primers given in Additional file 1: Table S12.
Disruption of $v d t X$ via repeat induced point mutation A different approach was taken to disrupt $v d t X$ after no gene replacements were identified from the homologous recombination approach. The process of repeat induced point (RIP) mutation during a sexual cross was employed. A partial $v d t X$ gene fragment was amplified using primers RIPF1 and RIPR2 and cloned into pPZPHygHindX [59] linearized with XbaI-EcoRV using Gibson assembly. The construct was then transformed into strain CBS 144490. One resultant transformant was crossed to strain CBS 101075 and progeny isolated as described previously [60]. The targeted regions of $v d t X$ in the progeny were amplified and sequenced with primers RIPseqF and RIPseqR to identify those progeny that had been heavily mutated by RIP.

\section{Metabolic profile analysis, compound isolation and characterization}

Czapek Yeast Extract Agar (CYA, 30 g sucrose, 5 g yeast extract, $1 \mathrm{~g} \mathrm{~K}_{2} \mathrm{HPO}_{4}, 0.3 \mathrm{~g} \mathrm{NaNO}_{3}, 0.05 \mathrm{~g} \mathrm{KCl}, 0.05 \mathrm{~g}$ $\mathrm{MgSO}_{4}, 0.01 \mathrm{~g} \mathrm{ZnSO}_{4}, 0.01 \mathrm{~g} \mathrm{FeSO}_{4}, 0.5 \mathrm{mg} \mathrm{CuSO}$, $15 \mathrm{~g}$ agar in $1 \mathrm{~L}$ ) was used for culturing of $P$. variotii strains and $A$. viridinutans for the accumulation of secondary metabolites. Agar cultures were chopped into small pieces and extracted with methanol with sonication. Crude extracts were filtered, dried and redissolved in methanol for LC-DAD-MS analysis.

LC-DAD-MS analyses were performed with an Agilent 1260 liquid chromatography (LC) system coupled to a diode array detector (DAD) and an Agilent 6130 quadrupole mass spectrum (MS) with an ESI source. For analytical purposes, a Kinetex $\mathrm{C} 18$ column $(2.6 \mu \mathrm{m}$, $2.1 \mathrm{~mm}$ i.d. $\times 100 \mathrm{~mm}$; Phenomenex) was used. The mobile phase gradient of eluent B (acetonitrile with $0.1 \%$ formic acid) started at $5 \%$ and gradually increased to $95 \%$ over $10 \mathrm{~min}$ at a flow rate of $0.75 \mathrm{ml} / \mathrm{min}$.

For compound isolation, $P$. variotii CYA culture of either wild-type strain or specific mutant was extracted twice with ethyl acetate/methanol (90:10). Crude extracts were dried in vacuo and then fractionated on a Reveleris flash chromatography system (Grace) using a dichloromethane/methanol gradient on a Reveleris HP silica flash cartridge. Fractions containing the target compound were combined for further purification using a semi-prep HPLC with a C18 column (Agilent, $5 \mu \mathrm{m}, 21.2 \times 150 \mathrm{~mm})$.

For structural characterization, nuclear magnetic resonance (NMR) spectra were collected for purified metabolites on a Bruker Avance IIIHD $500 \mathrm{MHz} / 600 \mathrm{MHz}$ NMR spectrometer. Chloroform- $d$, DMSO- $d_{6}$, and acetonitrile- $d_{3}$ were used as solvents. 
Electronic circular dichroism (ECD) spectra were recorded on a JASCO J-810 spectropolarimeter, with acetonitrile as solvent. The axial chirality of dimeric compounds isolated was determined by comparing with both the ECD spectrum of $(M)$-viriditoxin standard purchased from Sapphire Bioscience (Redfern, Australia) and published data [21].

\section{Generation of strains for the examination of fluorescently-tagged proteins}

The open reading frame of GFP was tagged immediately downstream of $v d t G$ and $v d t A$ via homologous recombination.

In the case of $v d t G$, a fragment corresponding to the end of the gene (omitting the stop codon) was amplified using primers 24GFP5F and 24GFP5R and GFP was amplified using primers 24GFPGFPF and 24GFPGFPR and cloned into plasmid pPZP-201BK linearized with EcoRI and HindIII using Gibson assembly. The HYG construct and $3^{\prime}$ flank of the gene was amplified with primers pairs 24GFPHYGF-24GFPHYGR and 24GFP3F24GFP3R off plasmids pMAI6 and PLAU17, respectively [38], and cloned into the EcoRI site of the previously generated plasmid to produce the final construct. This construct was transformed into $P$. variotii strain CBS 144490 using AtMT [27].

In the case of $v d t A$, a fragment corresponding to the end of the gene (omitting the stop codon) was amplified using primers 69GFP5F and 69GFP5R and the $3^{\prime}$ flank of the gene was amplified with primers 69GFP3F and 69GFP3R and cloned into plasmid pPZP-201BK linearized with EcoRI and HindIII using Gibson assembly. The combined GFP-HYG section of the construct previously generated to tag VdtG was amplified using 69GFPHYGF and 69GFPHYGR and cloned into the BamHI site of the previous construct. This construct was introduced into strain CBS 144490 by AtMT as described previously [27].

Constructs were generated for the purpose of resolving the subcellular localization of proteins within the cell, namely Erg11-mCherry to tag the endoplasmic reticulum and mCherry-SKL to tag the peroxisomes. Erg11 was amplified using primers Erg11F and Erg11R and mCherry was amplified using primers McherryErg11F and McherryErg11R off genomic DNA and plasmid mCherry-dspA [27], respectively, and cloned into the BglII site of plasmid PLAU53 which has been designed to express proteins under the expression of the actin promoter from Leptosphaeria maculans and confers resistance to G418 [38]. The mCherry isoform with the addition of sequence coding for the three amino acid sequence SKL was amplified using primers AP57 and AP58, and similarly cloned into PLAU53. The mCherry-SKL construct was transformed into the VdtA-GFP strain and the Erg11-mCherry construct was transformed into the VdtG-GFP strain.

Isolates were cultured PDB for 3 days, and then examined using a Leica DM6000 fluorescence microscope.

\section{Additional file}

Additional file 1. Supplementary data that feature Tables S1-S12 and Figures S1-S56.

\section{Acknowledgements}

We thank Mark Wilson (CSIRO, Australia) for providing the A. viridinutans strain and Allison van de Meene (University of Melbourne) for assistance with microscopy.

\section{Authors' contributions}

ASU, JH, Y-HC and Al designed the experiments. ASU performed the sequencing, sequence analysis, and genetic manipulations of $P$. variotii. JH performed the chemical purifications and metabolite characterizations. ASU, JH, Y-HC and $\mathrm{Al}$ analyzed and interpreted the data and results. ASU was the major contributor in writing the manuscript. JH, Y-HC and Al provided critical feedback and helped in writing the manuscript. All authors read and approved the final manuscript.

\section{Funding}

ASU received financial support from the Australasian Mycological Society and was supported by the RTS of the Australian government. Al and Y-HC received support as Future Fellows of the Australian Research Council, and Al was a Robert Lipp Plant Science Memorial Research Fellow of the University of Melbourne.

\section{Availability of data and materials}

The $A$. viridinutans genome sequencing raw reads and assembly are available from GenBank (BioProject PRJNA513223). NMR data are presented in the supplemental information. Strains are available from Al upon request.

Ethics approval and consent to participate

Not applicable.

Consent for publication

Not applicable.

\section{Competing interests}

The authors declare that they have no competing interests.

Received: 7 April 2019 Accepted: 12 June 2019

Published online: 01 July 2019

\section{References}

1. Lillehoj EB, Milburn MS. Viriditoxin production by Aspergillus viridi-nutans and related species. Appl Microbiol. 1973;26:202-5.

2. Samson RA, Houbraken J, Varga J, Frisvad JC. Polyphasic taxonomy of the heat resistant ascomycete genus Byssochlamys and its Paecilomyces anamorphs. Persoonia. 2009;22:14-27.

3. Jiu J, Mizuba S. Metabolic products from Spicaria divaricata NRRL 5771. J Antibiot. 1974;27:760-5

4. Frandsen RJN, Nielsen NJ, Maolanon N, Sørensen JC, Olsson S, Nielsen J, et al. The biosynthetic pathway for aurofusarin in Fusarium graminearum reveals a close link between the naphthoquinones and naphthopyrones. Mol Microbiol. 2006;61:1069-80.

5. Stewart M, Capon RJ, White JM, Lacey E, Tennant S, Gill JH, et al. Rugulotrosins A and B: two new antibacterial metabolites from an Australian isolate of a Penicillium sp. J Nat Prod. 2004;67:728-30. 
6. Hu J, Sarrami F, Li H, Zhang GZ, Stubbs KA, Lacey E, et al. Heterologous biosynthesis of elsinochrome $A$ sheds light on the formation of the photosensitive perylenequinone system. Chem Sci. 2019;10:1457-65.

7. Daub ME. Cercosporin, a photosensitizing toxin from Cercospora species. Phytopathology. 1982;72:370-4.

8. Stothers JB, Stoess A A. Confirmation of the polyketide origin of kotanin by incorporation of $\left[1,2-{ }^{13} \mathrm{C}_{2}\right]$ acetate. Can J Chem. 1988;66:2816-8.

9. Nozawa K, Seyea H, Nakajima S, Udagawa S-I, Kawai K-i. Studies on fungal products. Part 10. Isolation and structures of novel bicoumarins, desertorins A, B, and C, from Emericella desertorum. J Chem Soc Perkin Trans. 1987;1:1735-8.

10. Frandsen RJN, Schütt C, Lund BW, Staerk D, Nielsen J, Olsson S, Giese H. Two novel classes of enzymes are required for the biosynthesis of aurofusarin in Fusarium graminearum. J Biol Chem. 2011;286:10419-28.

11. Chooi YH, Zhang GZ, Hu J, Muria-Gonzalez MJ, Tran PN, Pettitt A, et al. Functional genomics-guided discovery of a light-activated phytotoxin in the wheat pathogen Parastagonospora nodorum via pathway activation. Environ Microbiol. 2017:19:1975-86.

12. Chen H, Lee MH, Daub ME, Chung KR. Molecular analysis of the cercosporin biosynthetic gene cluster in Cercospora nicotianae. Mol Microbiol. 2007;64:755-70

13. de Jonge R, Ebert MK, Huitt-Roehl CR, Pal P, Suttle JC, Spanner RE, et al. Gene cluster conservation provides insight into cercosporin biosynthesis and extends production to the genus Colletotrichum. Proc Natl Acad Sci USA. 2018;115:E5459-66.

14. Mazzaferro LS, Hüttel W, Fries A, Müller M. Cytochrome P450-catalyzed regio- and stereoselective phenol coupling of fungal natural products. Am Chem Soc. 2015;137:12289-95.

15. Wang J, Galgoci A, Kodali S, Herath KB, Jayasuriya H, Dorso K, et al. Discovery of a small molecule that inhibits cell division by blocking FtsZ, a novel therapeutic target of antibiotics. J Biol Chem. 2003;278:44424-8.

16. Foss MH, Eun YJ, Grove Cl, Pauw DA, Sorto NA, Rensvold JW, et al. Inhibitors of bacterial tubulin target bacterial membranes in vivo. MedChemComm. 2013:4:112-9.

17. Kundu S, Kim TH, Yoon JH, Shin HS, Lee J, Jung JH, et al. Viriditoxin regulates apoptosis and autophagy via mitotic catastrophe and microtubule formation in human prostate cancer cells. Int J Oncol. 2014;45:2331-40.

18. Park JH, Noh TH, Wang H, Kim ND, Jung JH. Viriditoxin induces $\mathrm{G} 2 / \mathrm{M}$ cell cycle arrest and apoptosis in A549 human lung cancer cells. Nat Prod Sci. 2015:21:282-8

19. Green P, Blaney B, Moore C, Connole M. Identification and preliminary evaluation of viriditoxin, a metabolite of Paecilomyces varioti, as an insecticide for sheep blowfly, Lucilia cuprina (Wied.). Gen Appl Entomol. 1989;21:33-7.

20. Silva MRO, Kawai K, Hosoe T, Takaki GMC, Gusmão NB, Fukushima K. Viriditoxin, an antibacterial substance produced by mangrove endophytic fungus Paecilomyces variotii. In: Méndez-Vilas A, editor. Microbial pathogens and strategies for combating them: science, technology and education, vol. 2. Badajoz: Formatex Research Center; 2013. p. 1406-11.

21. Suzuki K, Nozawa K, Nakajima S, Kawai K. Structure revision of mycotoxin, viriditoxin, and its derivatives. Chem Pharm Bull (Tokyo). 1990;38:3180-1.

22. Fürtges $L$, Obermaier $S$, Thiele $W$, Foegen $S$, Müller M. Diversity in fungal intermolecular phenol coupling of polyketides-regioselective laccasebased systems. ChemBioChem. 2019. https://doi.org/10.1002/cbic.20190 0041

23. Kawaguchi M, Ohshiro T, Toyoda M, Ohte S, Inokoshi J, Fujii I, et al. Discovery of a fungal multicopper oxidase that catalyzes the regioselective coupling of a tricyclic naphthopyranone to produce atropisomers. Angew Chem. 2018;57:5115-9.

24. Davin LB, Wang HB, Crowell AL, Bedgar DL, Martin DM, Sarkanen S, et al. Stereoselective bimolecular phenoxy radical coupling by an auxiliary (dirigent) protein without an active center. Science. 1997;275:362-6.

25. LaPlante SR, Fader LD, Fandrick KR, Fandrick DR, Hucke O, Kemper R, et al. Assessing atropisomer axial chirality in drug discovery and development. J Med Chem. 2011;54:7005-22.

26. Glunz PW. Recent encounters with atropisomerism in drug discovery. Bioorg Med Chem Lett. 2018;28:53-60.

27. Urquhart A, Mondo S, Mäkelä M, Hane J, Wiebenga A, He G, et al. Genomic and genetic insights into a cosmopolitan fungus, Paecilomyces variotii (Eurotiales). Front Microbiol. 2018;9:3058.
28. Malz S, Grell MN, Thrane C, Maier FJ, Rosager P, Felk A, et al. Identification of a gene cluster responsible for the biosynthesis of aurofusarin in the Fusarium graminearum species complex. Fungal Genet Biol. 2005;42:420-33.

29. Grigoriev IV, Nikitin R, Haridas S, Kuo A, Ohm R, Otillar R, et al. MycoCosm portal: gearing up for 1000 fungal genomes. Nucleic Acids Res. 2014:42:D699-704.

30. Szwalbe AJ, Williams K, Song Z, de Mattos-Shipley K, Vincent JL, Bailey $A M$, Willis CL, Cox RJ, Simpson TJ. Characterisation of the biosynthetic pathway to agnestins $A$ and $B$ reveals the reductive route to chrysophanol in fungi. Chem Sci. 2019;10:233-8.

31. Fox EM, Howlett BJ. Secondary metabolism: regulation and role in fungal biology. Curr Opin Microbiol. 2008;11:481-7.

32. Langfelder K, Jahn B, Gehringer H, Schmidt A, Wanner G, Brakhage AA. Identification of a polyketide synthase gene ( $p k s P$ ) of Aspergillus fumigatus involved in conidial pigment biosynthesis and virulence. Med Microbiol Immunol. 1998;187:79-89.

33. Tsai HF, Wheeler MH, Chang YC, Kwon-Chung KJ. A developmentally regulated gene cluster involved in conidial pigment biosynthesis in Aspergillus fumigatus. J Bacteriol. 1999;181:6469-77.

34. Heinekamp T, Thywissen A, Macheleidt J, Keller S, Valiante V, Brakhage AA. Aspergillus fumigatus melanins: interference with the host endocytosis pathway and impact on virulence. Front Microbiol. 2013;3:440.

35. Liu L, Zhang Z, Shao CL, Wang JL, Bai H, Wang CY. Bioinformatical analysis of the sequences, structures and functions of fungal polyketide synthase product template domains. Sci Rep. 2015;5:10463.

36. Selker EU, Garrett PW. DNA-sequence duplications trigger gene inactivation in Neurospora crassa. Proc Natl Acad Sci USA. 1988;85:6870-4.

37. Upadhyay S, Xu XP, Lowry D, Jackson JC, Roberson RW, Lin X. Subcellular compartmentalization and trafficking of the biosynthetic machinery for fungal melanin. Cell Rep. 2016;14:2511-8.

38. Idnurm A, Urquhart AS, Vummadi DR, Chang S, Van de Wouw AP, LópezRuiz FJ. Spontaneous and CRISPR/Cas9-induced mutation of the osmosensor histidine kinase of the canola pathogen Leptosphaeria maculans. Fungal Biol Biotechnol. 2017:4:12.

39. Bartoszewska M, Opaliński Ł, Veenhuis M, van der Klei IJ. The significance of peroxisomes in secondary metabolite biosynthesis in filamentous fungi. Biotechnol Lett. 2011;33:1921-31.

40. Maggio-Hall LA, Wilson RA, Keller NP. Fundamental contribution of $\beta$-oxidation to polyketide mycotoxin production in planta. Mol PlantMicrobe Interact. 2005;18:783-93.

41. Monosov EZ, Wenzel TJ, Luers GH, Heyman JA, Subramani S. Labeling of peroxisomes with green fluorescent protein in living P. pastoris cells. J Histochem Cytochem. 1996;44:581-9.

42. Calvo AM, Wilson RA, Bok JW, Keller NP. Relationship between secondary metabolism and fungal development. Microbiol Mol Biol Rev. 2002;66:447-59.

43. Chang PK, Yu JJ, Yu JH. afTT, a MFS transporter-encoding gene located in the aflatoxin gene cluster, does not have a significant role in aflatoxin secretion. Fungal Genet Biol. 2004;41:911-20.

44. Gardiner DM, Jarvis RS, Howlett BJ. The ABC transporter gene in the sirodesmin biosynthetic gene cluster of Leptosphaeria maculans is not essential for sirodesmin production but facilitates self-protection. Fungal Genet Biol. 2005;42:257-63.

45. Menke J, Weber J, Broz K, Kistler HC. Cellular development associated with induced mycotoxin synthesis in the filamentous fungus Fusarium graminearum. PloS One. 2013;8:e63077.

46. Chanda A, Roze LV, Kang S, Artymovich KA, Hicks GR, Raikhel NV, et al. A key role for vesicles in fungal secondary metabolism. Proc Natl Acad Sci USA. 2009;106:19533-8.

47. Boenisch MJ, Broz KL, Purvine SO, Chrisler WB, Nicora CD, Connolly LR, et al. Structural reorganization of the fungal endoplasmic reticulum upon induction of mycotoxin biosynthesis. Sci Rep. 2017;7:13.

48. Song JX, Zhai PF, Zhang YW, Zhang CY, Sang H, Han GZ, et al. The Aspergillus fumigatus damage resistance protein family coordinately regulates ergosterol biosynthesis and azole susceptibility. MBio. 2016;7:e01919-15.

49. Lai CY, Cronan JE. Isolation and characterization of $\beta$-ketoacyl-acyl carrier protein reductase ( $f a b G$ ) mutants of Escherichia coli and Salmonella enterica serovar typhimurium. J Bacteriol. 2004;186:1869-78. 
50. Pazmino DET, Dudek HM, Fraaije MW. Baeyer-Villiger monooxygenases: recent advances and future challenges. Curr Opin Chem Biol. 2010;14:138-44.

51. Wen Y, Hatabayashi H, Arai H, Kitamoto HK, Yabe K. Function of the cypX and moxY genes in aflatoxin biosynthesis in Aspergillus parasiticus. Appl Environ Microbiol. 2005;71:3192-8.

52. Südhof TC. Neuroligins and neurexins link synaptic function to cognitive disease. Nature. 2008;455:903-11.

53. Di Jeso B, Arvan P. Thyroglobulin from molecular and cellular biology to clinical endocrinology. Endocr Rev. 2016;37:2-36.

54. Antonian E. Recent advances in the purification, characterization and structure determination of lipases. Lipids. 1988;23:1101-6.

55. Ichtchenko K, Hata Y, Nguyen T, Ullrich B, Missler M, Moomaw C, et al. Neuroligin-1: a splice site-specific ligand for $\beta$-neurexins. Cell. 1995;81:435-43.

56. Hu J, Li H, Chooi YH. Fungal dirigent protein controls the stereoselectivity of multicopper oxidase-catalyzed phenol coupling in viriditoxin biosynthesis. J Am Chem Soc. 2019:141:8068-72.
57. Pitkin JW, Panaccione DG, Walton JD. A putative cyclic peptide efflux pump encoded by the TOXA gene of the plant-pathogenic fungus Cochliobolus carbonum. Microbiology. 1996;142:1557-65.

58. Zerbino DR, Birney E. Velvet: algorithms for de novo short read assembly using de Bruijn graphs. Genome Res. 2008;18:821-9.

59. Elliott CE, Howlett BJ. Overexpression of a 3-ketoacyl-CoA thiolase in Leptosphaeria maculans causes reduced pathogenicity on Brassica napus. Mol Plant-Microbe Interact. 2006;19:588-96.

60. Houbraken J, Varga J, Rico-Munoz E, Johnson S, Samson RA. Sexual reproduction as the cause of heat resistance in the food spoilage fungus Byssochlamys spectabilis (anamorph Paecilomyces variotii). Appl Environ Microbiol. 2008;74:1613-9.

\section{Publisher's Note}

Springer Nature remains neutral with regard to jurisdictional claims in published maps and institutional affiliations.
Ready to submit your research? Choose BMC and benefit from:

- fast, convenient online submission

- thorough peer review by experienced researchers in your field

- rapid publication on acceptance

- support for research data, including large and complex data types

- gold Open Access which fosters wider collaboration and increased citations

- maximum visibility for your research: over $100 \mathrm{M}$ website views per year

At BMC, research is always in progress.

Learn more biomedcentral.com/submissions 